\title{
Robust Converter Synchronization to a Weak Grid Through a DFT-based Method
}

\author{
António P. Martins and José C. Ferreira \\ Faculty of Engineering of the University of Porto \\ Department of Electrical and Computers Engineering \\ Rua Dr Roberto Frias, 4200-465 Porto (Portugal) \\ Phone +351 22 5081816, email: ajm@fe.up.pt, jcarfe@portugalmail.pt
}

\begin{abstract}
Phasor estimation and symmetrical components estimation is a fundamental task in power electronics converters control. Power conditioning systems like active filters, universal power flow controllers, static compensators, and dynamic voltage restorers, among other ones, need accurate synchronization circuits capable of dealing with very different grid voltage perturbations. Voltage sags and swells, harmonics, spikes and notches, frequency variation, phase steps, DC components and noise, are phenomena that can cause severe malfunction in the control or supervision circuits of such power systems. Additionally, some protection systems are sensitive to some of these parameters and can give wrong actions when they are not expected to. The DFT algorithm is one of best performers in phasor estimation, but is not perfect. The paper accurately describes the DFT errors under frequency variation, decaying DC components and harmonics, and analyzes the instantaneous phasor estimation, with absolute and recursive algorithms. Strong grid voltage perturbations, occurring in weak grids, especially in the grid connection of renewable energies, demand for a DFT-based robust estimation method. The proposed method, as demonstrated by extensive simulations, is capable of handling general grid voltage perturbations, imposing a compromise between dynamic response and accuracy.
\end{abstract}

\section{Keywords}

DFT algorithm, Phasor estimation, Synchronization methods, Power electronics, Converter control

\section{Introduction}

The Discrete Fourier Transform (DFT) has been widely used in the analysis of the fundamental component and harmonics of the electrical grid voltages and currents, [1]. The temporal information loss caused by the transformation is recovered by analyzing the signal in just one temporal window with duration of one or a multiple of the fundamental period of the analyzed waveform. The DFT method gives accurate results when the sampled period is equal to the fundamental period.
In case of input frequency variations there is a phase shift between the input and output signals, an error in the amplitude calculation as well as spectral leakage. With unknown input frequencies the signal components are projected to the presumed frequency components, multiples of the input frequency. During fast transients or faults, the grid voltage is characterized by being a non periodic signal containing fast oscillations, exponential decaying components and harmonics, among other possible disturbances. Fundamental component extraction by conventional DFT is affected by an important error due to the limited temporal resolution of the analyzed window.

The phase difference resulting from the difference between the presumed frequency and the real frequency can be compensated in different ways: by imposing that the analyzed interval should be equal to the grid period, [2], or by adding a phase offset to cancel the phase difference. The second method is preferable since it does not imply a change in the execution frequency of the digital algorithm that can be embedded in a control task with other algorithms that need to be executed at a fixed frequency. The so-called Smart Discrete Fourier Transform, [3], measures the input phasor signal and estimates the frequency with high precision, superior to the conventional DFT, showing robustness and being implemented in a recursive manner. The estimation precision is robust in the presence of noise and is higher if it is considered high order harmonics but implying a more complex algorithm and pre-filtering, [4].

Different methods have been proposed to allow the DFT algorithm to deal with variable frequency input signals, exponential decaying components and noise immunity. Adaptive variation of the temporal window, adaptive change of the sampling frequency, phase and amplitude correction and input data modification are the main proposed methods to increase the DFT algorithm performance. 
Input low-pass anti-aliasing filters can eliminate the high frequency components but can not remove decaying dc components and reject low frequency components. Under these conditions, the DFT based phasor estimation is more difficult and slower and affects the performance of converters synchronization and digital relaying.

Absolute and recursive DFT modified algorithms can be applied to some of the above mentioned non nominal operating conditions. However, a more realistic list of abnormal field operating conditions includes:

- Amplitude variation (sags and swells). If voltage swells are important because they can cause serious damage in electrical machines and transformers, voltage sags are becoming more demanding since there is a need to maintain some important grid connected system in operation even under high amplitude sags, [5].

- Harmonics. The presence of high power non linear loads, deregulation rules and increased power flow allowed the increase of harmonics presence in the grid voltage.

- Spikes and notches. Caused by power devices switching and capacitors commutation they can severely affect zero crossing detection and generate low frequency harmonics.

- Frequency variation (step and continuous). High active power variations, generators failure and power transfers between large connected areas can cause frequency variations. As voltage sags, frequency deviations from the nominal value are imposing new and demanding conditions in the new energy generation era.

- Phase steps. Connecting and disconnecting large loads, especially in weak grids, are the main origin of phase steps occurrence. Having an extremely large frequency spectrum they cause important transient phenomena as in amplitude measurement or frequency estimation as in control systems.

- Exponential decaying components. Generated by different fault types or grid connection of high power electrical machines they constitute with phase steps the more important disturbances that appear in a grid voltage system.

- Noise. Always present and generated by very different sources.

Phasor estimation and symmetrical components calculation for power conditioning converters control under different grid voltage perturbations are the parameters to be analyzed in the paper. Performance optimization for the all conditions should be a compromise.

\section{Phasor Detection with the DFT Algorithm}

Apart from noise and harmonics, to be considered later, the main components of a voltage signal from the phasor detection point of view can be expressed as:

$$
x(t)=X \cos (\omega t+\phi)+A e^{-t / \tau}
$$

where $A$ is the initial amplitude of a decaying DC component, $\tau$ its time constant, $X$ is the amplitude of the voltage signal, $\omega$ the fundamental frequency, and $\phi$ the initial phase angle.

Assume that $x(t)$ is sampled at a sampling rate $f_{s}$ :

$$
f_{S}=\frac{1}{T_{S}}=f_{o} N
$$

Being $f_{o}$ the nominal frequency and $N$ the number of samples per fundamental nominal period, the sampling produces a data sequence $x\left(k T_{s}\right)$, or $x(k)$ :

$$
x(k)=X \cos \left(\omega \frac{k}{f_{o} N}+\phi\right)+A e^{-k T_{S} / \tau}
$$

Using a phasor representation with

$$
\bar{x}=X e^{j \phi}=X \cos \phi+j X \sin \phi
$$

The signal $x(k)$ can be represented by

$$
x(k)=\frac{\bar{x} e^{j \omega t_{k}}+\bar{x}^{*} e^{-j \omega t_{k}}}{2}+A e^{-k T_{S} / \tau}
$$

where $*$ denotes complex conjugate. The fundamental frequency component, $X_{1}$, (at instant $k$ and with nominal frequency, $f=f_{o}$ ) given by the DFT algorithm is

$$
X_{1}(k)=\frac{2}{N} \sum_{i=0}^{N-1} x(k+i-N) e^{-j \frac{2 \pi}{N} i}
$$

It is important to note that at instant $k$, the data window used to compute the DFT goes from $k-N$ to $k-1$. Taken frequency variation, $f=f_{o}+\Delta f$, into consideration and substituting the signal phasor representation in the DFT expression, the fundamental component is

$$
\begin{aligned}
& X_{1}(k)=\frac{\bar{x}}{N} \sum_{i=0}^{N-1} e^{j 2 \pi\left(f_{O}+\Delta f\right) \frac{k+i-N}{f_{O} N}} \cdot e^{-j \frac{2 \pi}{N} i} \\
& +\frac{\bar{x}^{*}}{N} \sum_{i=0}^{N-1} e^{-j 2 \pi\left(f_{O}+\Delta f\right) \frac{k+i-N}{f_{O} N}} \cdot e^{-j \frac{2 \pi}{N} i} \\
& +\frac{2 A}{N} \sum_{i=0}^{N-1} e^{-\frac{k+i-N}{f_{O} N \tau}} \cdot e^{-j \frac{2 \pi}{N} i}
\end{aligned}
$$

With some algebraic manipulation, the expression can be given by

$$
\begin{aligned}
& X_{1}(k)=\frac{\bar{x}}{N} e^{j \frac{2 \pi}{N} k} \cdot \frac{\sin \left(N \theta_{1}\right)}{\sin \left(\theta_{1}\right)} \cdot e^{j \frac{\pi}{N} \frac{\Delta f}{f_{O}}(2 k-N-1)} \\
& +\frac{\bar{x}^{*}}{N} e^{-j \frac{2 \pi}{N}(k-1)} \cdot \frac{\sin \left(N \theta_{2}\right)}{\sin \left(\theta_{2}\right)} \cdot e^{-j \frac{\pi}{N} \frac{\Delta f}{f_{o}}(2 k-N-1)} \\
& -\frac{2 A}{N} \cdot \frac{e^{\frac{1}{f_{O} \tau}}-1}{e^{-\frac{1}{f_{O} N \tau}-j \frac{2 \pi}{N}}-1} \cdot e^{-\frac{k}{f_{O} N \tau}}
\end{aligned}
$$


The frequency deviation dependent angles $\theta_{1}$ and $\theta_{2}$ are given by

$$
\theta_{1}=\frac{\pi}{N} \frac{\Delta f}{f_{o}} ; \theta_{2}=\frac{\pi}{N}\left(2+\frac{\Delta f}{f_{o}}\right)
$$

Harmonics presence in the grid voltage when there is a frequency deviation creates additional errors. It can be shown that the existence of $m$ harmonics causes an error in the fundamental component that is given by:

$$
\Delta X_{1}(k)=\frac{1}{N} \sum_{\substack{h=-m \\ h \neq-1,+1}}^{m} X_{h} e^{j \phi h} \cdot \frac{\sin \left(N \theta_{3}\right)}{\sin \left(\theta_{3}\right)} \cdot e^{j \theta_{4}}
$$

where

and

$$
\begin{gathered}
\theta_{3}=\frac{\pi}{N}\left(h-1+h \frac{\Delta f}{f_{o}}\right) \\
\theta_{4}=\frac{\pi}{N}\left[h\left(1+\frac{\Delta f}{f_{o}}\right)(2 k-N-1)-N+1\right] .
\end{gathered}
$$

Eq. (8) and (10) clearly show the behaviour of the DFT algorithm under abnormal conditions. In what respects to frequency deviation the resulting fundamental component has two types of errors: amplitude and phase. The positive direction rotating phasor presents a frequency deviation dependent amplitude and phase; the negative direction rotating phasor has also variable amplitude and rotates at a double frequency. Harmonics have a similar but much smaller contribution to the referred errors. The presence of an exponential decay component introduces a complex error, very unfavourable in phasor detection.

The DFT algorithm with these two conditions, variable frequency and exponential component, can be used if the resulting errors are correctly handled.

\section{A. Estimation under Variable Frequency}

There are some approaches to variable frequency operation: analytical correction based on absolute DFT calculation ([3], [6]); recursive DFT algorithm ([2], [7], [8]); high frequency filtering ([9]). In [6], frequency estimation is based on the phase variation given by two DFT calculations and approximate expressions so containing a frequency deviation dependent error. The method presented in [3] is simple in ideal conditions and can be recursive, but is susceptible to harmonics; its consideration highly increases the method complexity. Recursive DFT approaches share the same initial simplicity. When dealing with variable frequency the correction methods are very different, essentially depending on the measurement purpose. In [7] it is analyzed only phase correction, not amplitude, which is important for phasor estimation. Sampling frequency variation, proposed in [2], is not a feasible method; also, linear correction of the measured phase presents good results but is not tested in all conditions. Recursive DFT calculation with phase and amplitude correction, as presented in [8], works well. However, DC decaying components cause significant perturbations in phasor estimation. Filtering the high frequency components present in the amplitude and phase values returned by the DFT is limited to very small frequency deviations [9]. Also, filtering introduces an additional delay in the phasor estimation.

In general, it can not be guaranteed the absence of even harmonics, so any method based on the half cycle DFT is not considered. The relative slower dynamics of the full cycle DFT must be assumed.

\section{B. Decaying DC Component Compensation}

Accurate elimination of the exponentially decaying DC component from the fundamental phasor calculated by the DFT is treated by different methods: mimic filter [10], input data correction ([11], [12]) and analytical calculation with variable data window ([13]).

The mimic filter just uses an average value of the presumed decay component time constant, amplifies high frequency components and introduces a phase advance in the fundamental component, so making it unsuitable for accurate instantaneous phasor detection. Also, operation under variable frequency introduces amplitude errors. Input data correction, so eliminating the decay component, can be made just one cycle after the occurrence of a fault. Naturally, there is a need to detect the fault; the method is tailored for fault occurrence. The correct fundamental component phasor is only obtained after $N$ samples ([12]) or $N+4$ samples ([14]). Of course, this is much better than the simple DFT algorithm that originates an amplitude overshoot and settling time dependent on the time constant but also is done with high complex calculations. In practical applications this complexity results in two very important aspects: execution time and run time errors, due to trigonometric operations and possible divide by zero operations or square root calculations, respectively. The variable data window method in ([13]) is not so complex and is also fast but does not consider frequency deviation. Additionally, its main algorithm is tailored for fault detection, not for permanent operation like grid voltage feature extraction or control purposes like synchronization or current control.

\section{General Purpose DFT-Based Method}

The proposed method is focussed in power converters control and protection. It is intended for operating under all input voltage conditions including the above referred ones. The most demanding are the decaying DC components and the frequency variations. With these conditions present it was made an extensive study on the performance of a DFT-based method for operating under a general and unknown electrical environment.

Different criteria have been used to assess the performance of a particular method. Operation in all mandatory conditions must be a compromise between dynamics and precision: dynamics in order to efficiently control the converter currents and correctly measure the amplitude value with no over or undershoots; precision to 
accurately compute the parameters of interest and the control algorithms output values.

\section{A. General Purpose Method}

An on line DFT-based phasor estimation method with decaying DC component correction and frequency deviation compensation is presented. Among the different possibilities for minimizing the effects of a decaying DC component in DFT fundamental component phasor estimation, the partial sums approach will be used. Being excellent in the case of a fault occurrence, it deteriorates the DFT performance under other transients like voltage sags and swells, and phase steps. Also, it should be considered that a frequency deviation creates an error in the corrected data, so affecting its performance. The partial sums are defined by:

$$
\begin{gathered}
P S_{1}=x(1)+x(3)+\ldots+x(N-1) \\
P S_{2}=x(2)+x(4)+\ldots+x(N)
\end{gathered}
$$

In the absence of a decaying DC component and with nominal frequency the two sums are zero and the acquired data are not changed. Frequency deviation introduces an error that must be considered in real-time phasor estimation. The partial sums then result in:

$$
\begin{gathered}
P S_{1}(k)=A \cdot \frac{b\left(b^{N}-1\right)}{b^{2}-1}+e_{1}(k) \\
P S_{2}(k)=A \cdot \frac{b^{2}\left(b^{N}-1\right)}{b^{2}-1}+e_{2}(k)
\end{gathered}
$$

where $b=\exp \left(-1 /\left(f_{o} N \tau\right)\right)$ and the errors are given by:

$$
\begin{gathered}
e_{1}(k)=X \sin \left[\frac{2 \pi}{N}\left(1+\Delta f / f_{o}\right)(k-N / 2)\right] \cdot c \\
e_{2}(k)=X \sin \left[\frac{2 \pi}{N}\left(1+\Delta f / f_{o}\right)(k-N / 2+1)\right] \cdot c
\end{gathered}
$$

with

$$
c=\frac{\sin \left[\pi\left(1+\Delta f / f_{o}\right)\right]}{\sin \left[2 \pi / N\left(1+\Delta f / f_{o}\right)\right]} .
$$

The errors are dependent on the fundamental component amplitude, $X$, the frequency deviation, $\Delta f$, the sampling frequency, $N f_{o}$, and the actual instant, $k$. At each sampling instant, the partial sums must be calculated according to (14) and (15); then $A$ and $b$ are determined by simple algebra, ([12]).

The data correction made at each sampling instant by the DC component compensation algorithm does not allow using the recursive DFT method. The approach to deal with the frequency deviation is based on the method presented in [8] but modified to the absolute version of the DFT algorithm. Some increase in the computational needs is the consequence of a more broad-spectrum algorithm.

\section{B. Simulation Results}

As referred in the Introduction, the grid voltage is subjected to very different phenomena. So, a phasor estimation method must be tested against conditions like the ones that will be faced in field operation. The simulated conditions can be divided in three categories: voltage perturbations, frequency deviations and harmonics and noise rejection. In the voltage perturbations category it is considered voltage sags and swells, low frequency AC voltage superposition and decaying DC components; in the frequency deviations it is analyzed the behaviour under step and continuous frequency variations and phase steps; random noise and low frequency harmonics presence in association with frequency estimation precision are considered in the last group. The main simulation parameters are: the nominal voltage is 1 p.u., the nominal frequency is $50 \mathrm{~Hz}$, with 64 samples per period.

Due to a deregulated market and a high penetration level of renewable power sources strong voltage perturbations are expected in the near future. The amplitude voltage perturbations are presented in Fig. 1, 2, 3 and 4. For all the four conditions, several tests have been made. In all of them, the dynamics of the transient response is dependent on the instant when the perturbation occurred. The presented ones show the worst situations. Fig. 1 shows between $t=0.3 \mathrm{~s}$ and $t=0.4 \mathrm{~s}$, the collapse of the voltage down to $20 \%$, the instantaneous voltage estimation, the amplitude and phase detection, and the instantaneous frequency deviation. Fig. 2 shows the same waveforms but for a voltage swell of $150 \%$, between $t=0.5 \mathrm{~s}$ and $t=0.6 \mathrm{~s}$. Low frequency $\mathrm{AC}$ voltage superposition is analysed in Fig. 3, with an amplitude of $30 \%$ and a frequency of $10 \mathrm{~Hz}$. Exponentially decaying DC components are caused by several fault types including short circuits and overloads; Fig. 4 shows such an example: at $t=0.185 \mathrm{~s}$ the voltage collapses to zero; then, at $\mathrm{t}=0.25 \mathrm{~s}$ the voltage raises again, with an exponential decaying component with $A=1$ p.u. and a time constant of $40 \mathrm{~ms}$.
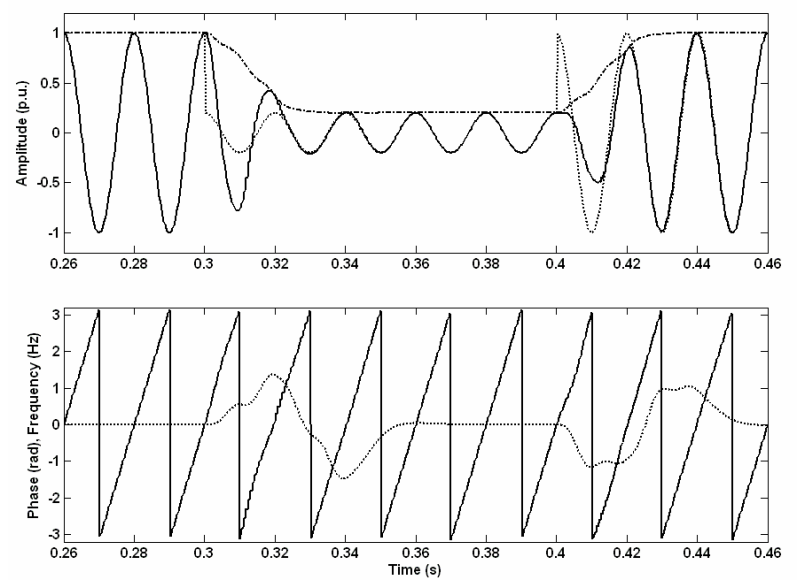

Fig. 1. DFT based phasor estimation when the grid is subjected to a $80 \%$ voltage sag. Upper traces: input voltage: $\cdots \cdot$; instantaneous estimation: — ; amplitude detection: ${ }^{-\cdots-}$. Lower traces: phase estimation: - ; frequency deviation: $\cdots$. 

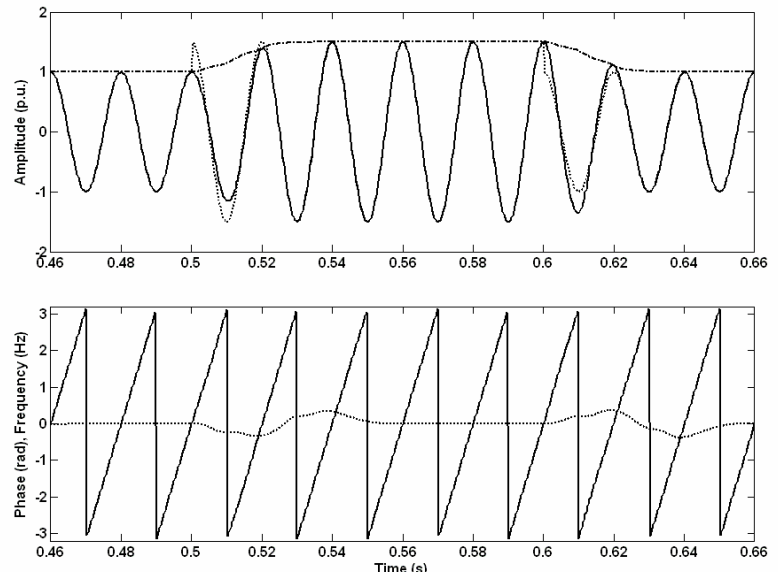

Fig. 2. DFT based phasor estimation when subjected to a $150 \%$ voltage swell. Upper traces: input voltage: …. ; instantaneous

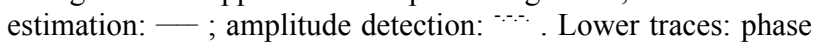
estimation: - ; frequency deviation: $\cdots$. .
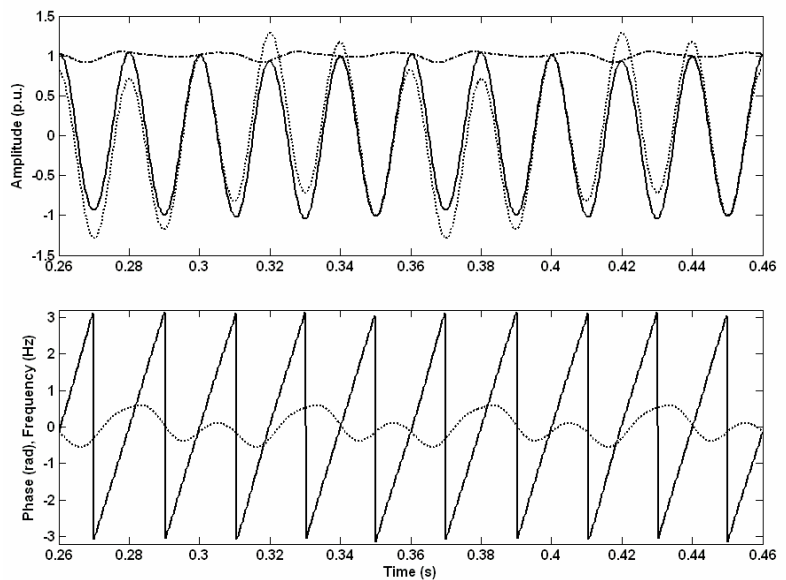

Fig. 3. Fluctuating $\mathrm{AC}$ voltage rejection. Upper traces: input voltage: … ; instantaneous estimation: - ; amplitude detection: $\cdots$. Lower traces: phase estimation: — ; frequency deviation: $\cdots$.
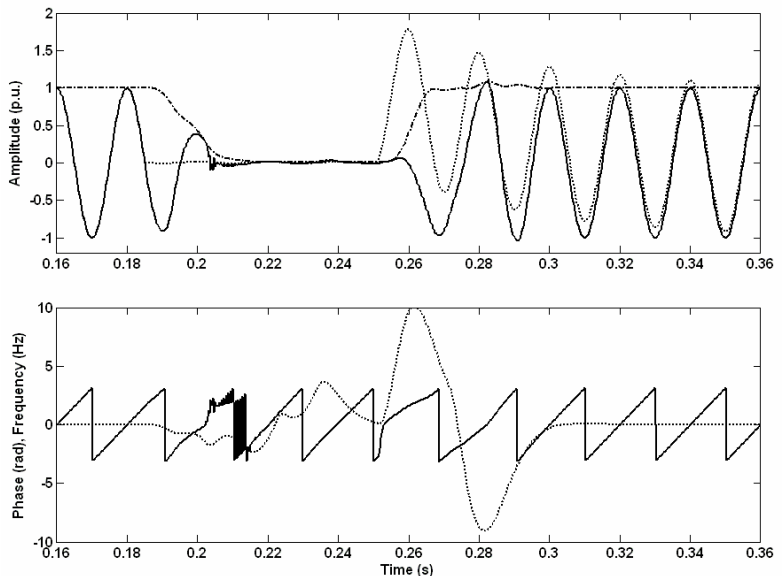

Fig. 4. Decaying DC component rejection. Upper traces: input voltage: … ; instantaneous estimation: - ; amplitude detection: $\cdots$. Lower traces: phase estimation: - ; frequency deviation: $\cdots$.
Voltage sags and swells have one and a half cycle time response; low frequency AC voltage fluctuation is efficiently rejected but introduces small frequency oscillations. Decaying DC components generate a phase and frequency perturbation but are correctly handled in amplitude.

Frequency perturbations come from generationconsumption unbalance in static and dynamic conditions. Frequency deviation steps are not usual in strong grids but can occur in weak systems; continuous frequency deviations and phase steps are much more common. Three conditions are presented in Fig. 5, 6 and 7.

In Fig. 5, frequency goes from $50 \mathrm{~Hz}$ to $47 \mathrm{~Hz}$ between $t=0.3 \mathrm{~s}$ and $t=0.4 \mathrm{~s}$, and from $50 \mathrm{~Hz}$ to $53 \mathrm{~Hz}$ between $t=0.5 \mathrm{~s}$ and $t=0.6 \mathrm{~s}$. With a time response of two cycles the frequency is correctly tracked with no amplitude errors. Continuous frequency tracking is showed in Fig. 6 with the frequency varying between $48.5 \mathrm{~Hz}$ and $50.5 \mathrm{~Hz}$ during the interval $t=[0.34 \mathrm{~s}, 0.74 \mathrm{~s}]$.

Phase steps cause severe perturbations at different levels; Fig. 7 shows the instantaneous phasor estimation, amplitude estimation and frequency deviation when the input voltage is subjected to a positive 60 degrees phase step at $t=0.225 \mathrm{~s}$, and a negative 60 degrees phase step at $t=0.325 \mathrm{~s}$. As in amplitude detection as in frequency estimation the perturbations are important but are correctly handled by the DFT based estimation method.

Different noise types generated by electromagnetic interference, digital circuits or power electronics converters are always present in the acquired signals. Also, low frequency harmonics due to non linear loads or saturated magnetic circuits are common in the grid voltage. Fig. 8 shows the noise rejection capability of the presented DFT based method when the processed signal is noise corrupted with a signal to noise ratio as low as 20 $\mathrm{dB}$ between $t=0.2 \mathrm{~s}$ and $t=0.3 \mathrm{~s}$.

The DFT algorithm is immune to harmonics, but only at nominal frequency; when there is a frequency deviation from the nominal value the presented DFT based method is also capable of maintaining harmonics immunity as is demonstrated in Fig. 9. Between $t=0.3 \mathrm{~s}$ and $t=0.4 \mathrm{~s}$, the frequency goes to $48 \mathrm{~Hz}$ and the signal contains a $20 \%$ amplitude $5^{\text {th }}$ harmonic and a $10 \%$ amplitude $7^{\text {th }}$ harmonic. There is no noticeable perturbation in the amplitude detection or in the instantaneous fundamental component estimation. For system protection and control functions, it is needed precision in frequency and amplitude estimation.

Fig. 10 and 11 present the algorithm precision at two frequencies, $47 \mathrm{~Hz}$ and $50 \mathrm{~Hz}$, when the processed signal contains a $20 \%$ amplitude $5^{\text {th }}$ harmonic and a $10 \%$ amplitude $7^{\text {th }}$ harmonic, and a signal to noise ratio of 40 $\mathrm{dB}$. In the two cases, the frequency estimation precision is better than $0.1 \%$ and the amplitude detection is better than $1 \%$. 

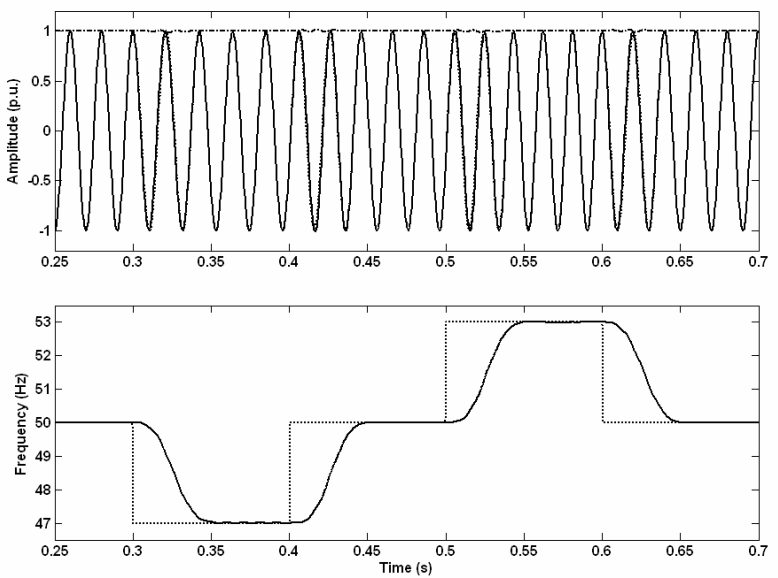

Fig. 5. Phasor estimation under step frequency variation. Upper traces: input voltage: $\cdots . .$. ; instantaneous estimation: amplitude detection: ${ }^{\cdots-\cdots}$. Lower traces: input frequency: $\cdots$; frequency estimation: - .

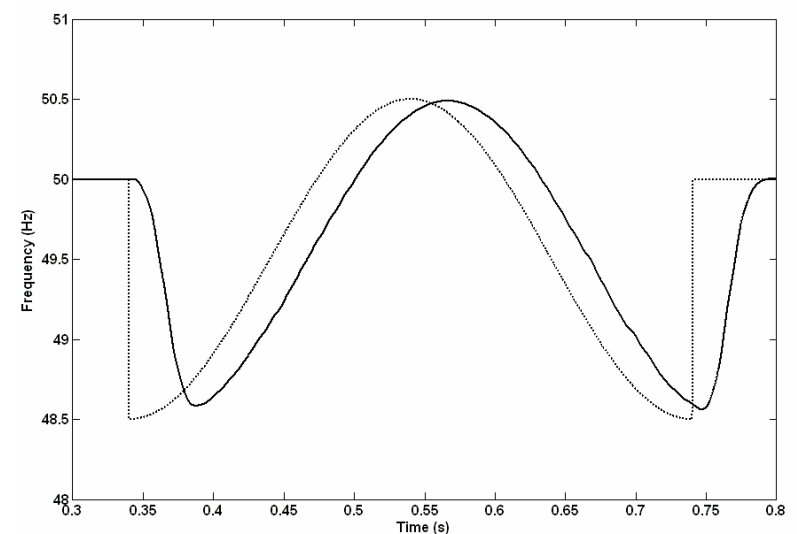

Fig. 6. Phasor estimation under step $(50 \mathrm{~Hz}$ to $49,5 \mathrm{~Hz})$ continuous $(+/-1 \mathrm{~Hz})$ frequency variation. Traces: input frequency: $\cdots$; frequency estimation: - .
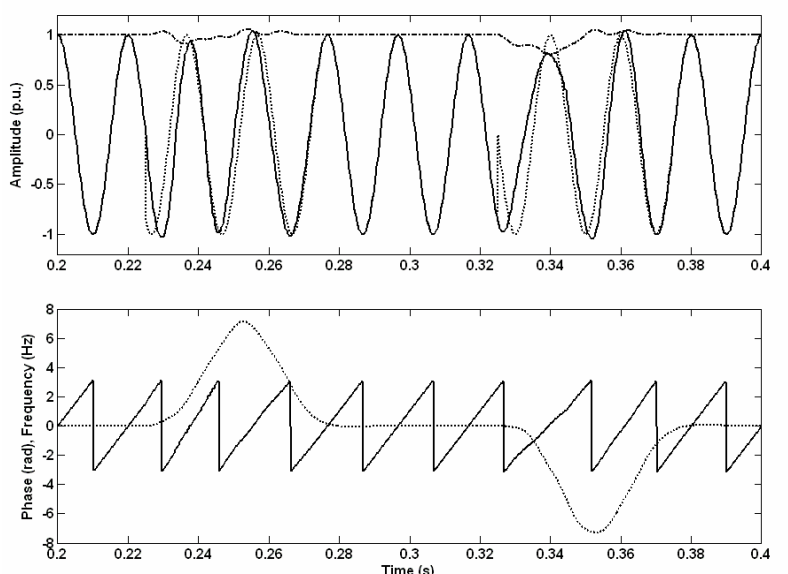

Fig. 7. Positive and negative phase steps. Upper traces: input voltage: $\cdots . .$. ; instantaneous estimation: - ; amplitude detection: ${ }^{\cdots-\cdots}$. Lower traces: phase estimation: — ; frequency deviation: $\cdots \cdot$.
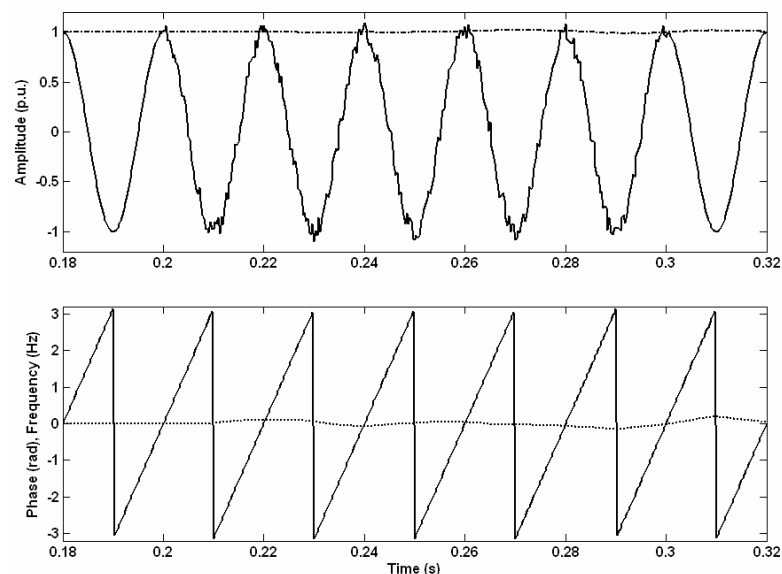

Fig. 8. Random noise rejection with nominal frequency and a $S / N$ ratio of $20 \mathrm{~dB}$. Upper traces: input voltage: - ; amplitude detection: ${ }^{\cdots \cdots}$. Lower traces: phase estimation: - - frequency deviation: $\cdots \cdot$.
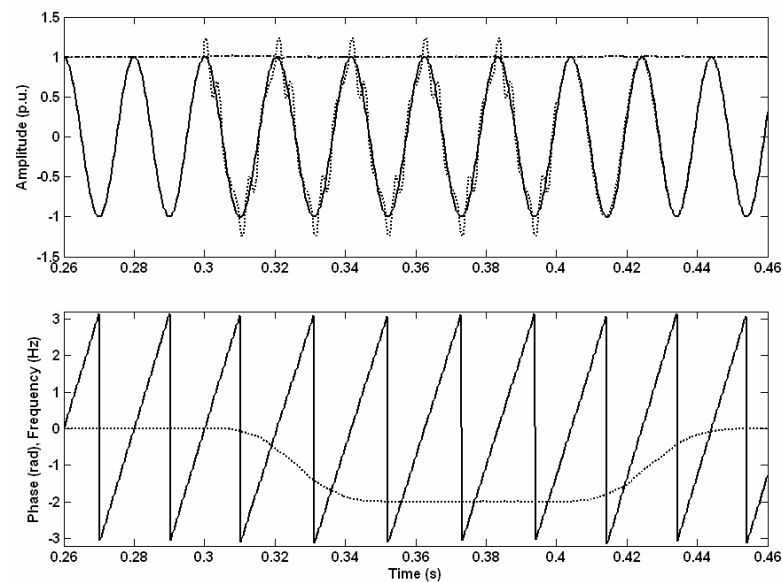

Fig. 9. Harmonics rejection under frequency deviation. Upper traces: input voltage: $\cdots \cdot$; instantaneous estimation: - ; amplitude detection: ${ }^{\cdots \cdots}$. Lower traces: phase estimation: - ; frequency deviation: $\cdots \cdot$.
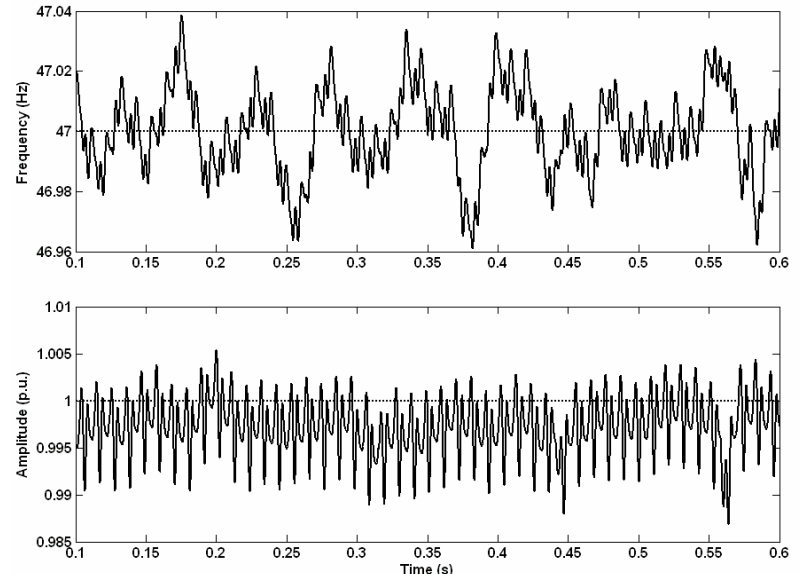

Fig. 10. Frequency and amplitude estimation precision with harmonics and noise presence at $f=47 \mathrm{~Hz}$. Upper traces: input frequency: $\cdots . \cdots$; frequency estimation: - . Lower traces: input amplitude: $\cdots \cdot$; amplitude estimation: - . 

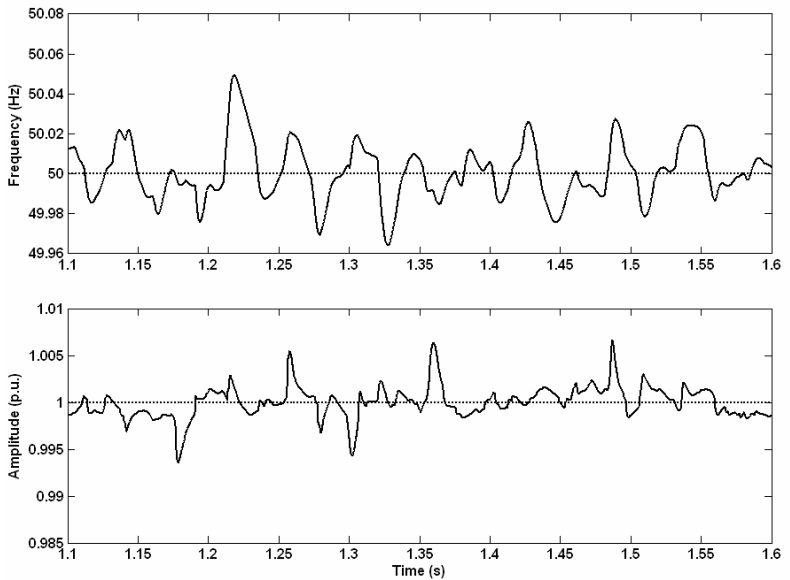

Fig. 11. Frequency and amplitude estimation precision with harmonics and noise presence at $f=50 \mathrm{~Hz}$. Upper traces: input frequency: $\cdots$; frequency estimation: - . Lower traces: input amplitude: $\cdots \cdot$; amplitude estimation: - .

All the presented results are dependent on the imposed conditions; some can be managed in real experimental implementations like noise level and low frequency harmonic distortion. The others are uncontrollable: sags, swells, AC fluctuation, DC decaying components, frequency deviations and phase steps will occur in an unpredictable way and level. Such conditions are general and very difficult to satisfy. Any phasor estimation method should be prepared to deal with them guaranteeing appropriate dynamics, stability, precision and robustness; the presented method does.

\section{Conclusion}

High power electronics converters control and protection need fast phasor estimation with instantaneous amplitude and phase calculation. Also, accurate frequency and amplitude estimation is needed for power systems stability analysis. Phenomena like high amplitude voltage sags and swells, decaying DC components, phase and frequency deviations, harmonics and noise are becoming more frequent and more intense, especially in weak grids. The corrupted voltage is difficult to manage in all conditions. In this paper, the DFT algorithm recognized robustness is extended to handle this new and more demanding grid voltage behaviour. The main errors caused by large frequency deviations and DC decaying components occurring in the DFT algorithm are recognized and analyzed, and the associated corrections to deal with the referred parameters are presented. The results, obtained in very unfavourable conditions, shown that it is needed a careful signal conditioning and a computationally powerful control platform in order to obtain fast dynamics and high accuracy.

\section{References}

[1] A. G. Phadke, J. S. Thorp, M. G. Adamiak, "A New Measurement Technique for Tracking Voltage Phasors, Local System Frequency, and Rate of Change of Frequency", IEEE Transactions on Power Apparatus and Systems, vol. PAS-102, no 5, pp. 1025-1038, May 1983.
[2] B. P. McGrath, D. G. Holmes, J. Galloway, "Improved Power Converter Line Synchronisation Using an Adaptive Discrete Fourier Transform (DFT)" Proceedings of the IEEE Power Electronics Specialists Conference, Cairns, Queensland, Australia, vol. 2, pp. 821-826, June 2002.

[3] J.-Z. Yang, C.-W. Liu, "A Precise Calculation of Power System Frequency and Phasor", IEEE Transactions on Power Delivery, vol. 15, n 2, pp. 494-499, April 2000.

[4] J.-Z. Yang, C.-W. Liu, "A Precise Calculation of Power System Frequency", IEEE Transactions on Power Delivery, vol. 16, n 3, pp. 361-366, July 2001.

[5] C. Jauch, P. Sorensen, B. Bak-Jensen, "International Review of Grid Connection Requirements for Wind Turbines", Proceedings of the Nordic Wind Power Conference, Chalmers University of Technology, Sweden, March 2004.

[6] D. Hart, D. Novosel, Y. Hu, B. Smith, M. Egolf, “A New Frequency Tracking and Phasor Estimation Algorithm for Generator Protection", IEEE Transactions on Power Delivery, vol. 12, nº 3, pp. 1064-1073, July 1997.

[7] T. Funaki, K. Matsuura, S. Tanaka, "Error Correction for Phase Detection by Recursive Algorithm Real Time DFT", Electrical Engineering in Japan, vol. 141, $\mathrm{n}^{\circ} 1$, pp. 8-17, 2002.

[8] M. Wang, Y. Sun, "A Practical, Precise Method for Frequency Tracking and Phasor Estimation", IEEE Transactions on Power Delivery, vol. 19, $n^{\circ}$ 4, pp. 15471552, October 2004.

[9] K. Nakano, Y. Ota, H. Ukai, K. Nakamura, H. Fujita, "Frequency Detection Method Based on Recursive DFT Algorithm", Proceedings of the $14^{\text {th }}$ Power Systems Computation Conference (PSCC), Sevilla, Spain, Session 1, Paper 5, June 2002.

[10] G. Benmouyal, "Removal of DC-offset in Current Waveforms Using Digital Mimic Filtering", IEEE Transactions on Power Delivery, vol. 10, $\mathrm{n}^{\circ} 2$, pp. 621630, April 1995,

[11] J.-C. Gu, S.-L. Yu, "Removal of DC Offset in Current and Voltage Signals Using a Novel Fourier Filter Algorithm" IEEE Transactions on Power Delivery, vol. $15, \mathrm{n}^{\mathrm{o}} 1$, pp. 73-79, January 2000.

[12] Y. Guo, M. Kezunovic, D. Chen, "Simplified Algorithms for Removal of the Effect of Exponentially Decaying DC-Offset on the Fourier Algorithm", IEEE Transactions on Power Delivery, vol. 18, n 3, pp. 711-717, July 2003.

[13] C.-S. Chen, C.-W. Liu, J.-Z. Yang, “A DC Offset Removal Scheme with a Variable Data Window for Digital Relaying", Proceedings of the Power Systems and Communications Infrastructures for the Future Conference, Beijing, September 2002.

[14] J.-Z. Yang C.-W. Liu, "Complete Elimination of DC Offset in Current Signals for Relaying Applications", Proceedings of the IEEE Power Engineering Society Winter Meeting, vol. 3, pp. 1933-1038, Singapore, January 2000. 